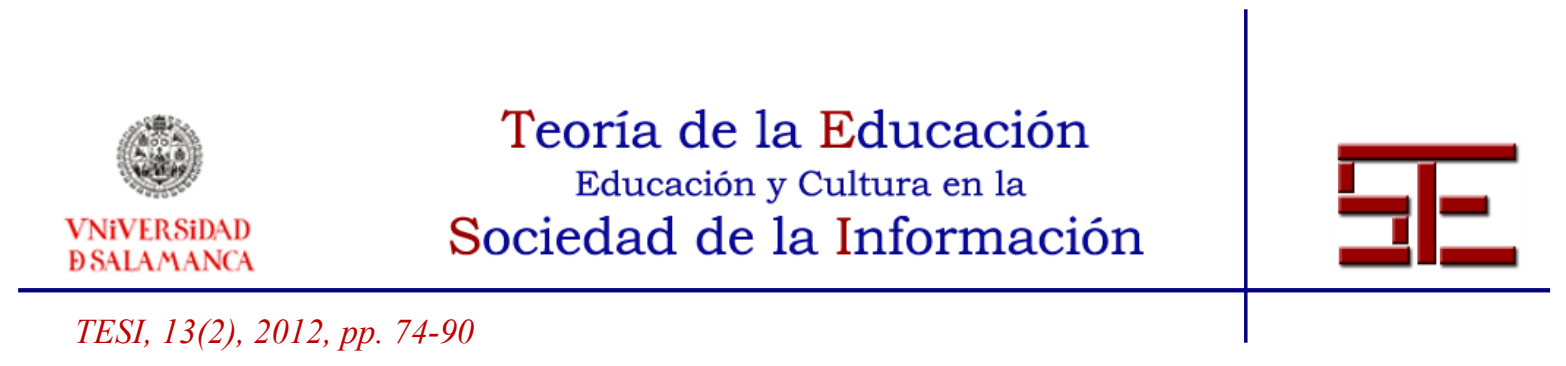

\title{
LA ROBÓTICA EDUCATIVA, UNA HERRAMIENTA PARA LA ENSEÑANZA-APRENDIZAJE DE LAS CIENCIAS Y LAS TECNOLOGÍAS
}

Resumen: En este artículo se presenta y analiza la robótica educativa como una herramienta de apoyo al proceso de enseñanza-aprendizaje, a nivel de pre-media, orientada principalmente a asignaturas complejas como la matemática, física e informática, entre otras. El estudio se limita a los colegios secundarios de la Provincia de Chiriquí, República de Panamá; se tomó una muestra de seis colegios de la provincia y por cada colegio participaron tanto estudiantes como docentes. El objetivo principal del proyecto fue demostrar como la robótica aplicada a la educación, facilita y motiva la enseñanza-aprendizaje de las ciencias y las tecnologías. Los resultados demostraron que la robótica se puede convertir en una herramienta excelente para comprender conceptos abstractos y complejos en asignaturas del área de las ciencias y las tecnologías; así como también permite desarrollar competencias básicas tales como trabajar en equipo.

Palabras clave: robótica educativa: proceso de enseñanza-aprendizaje; competencias; kits robóticos.

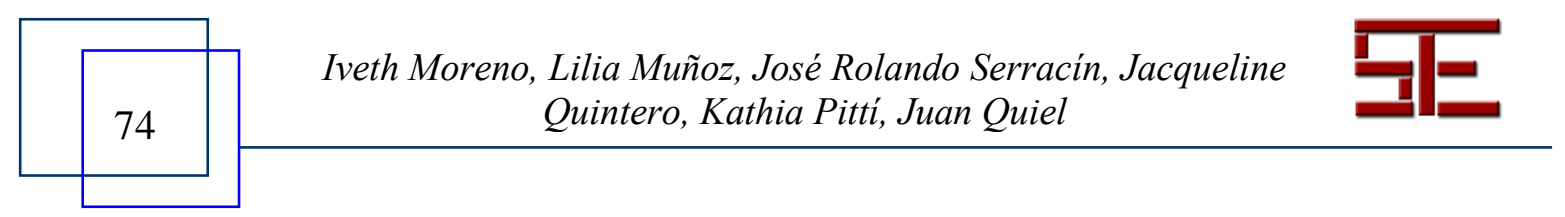




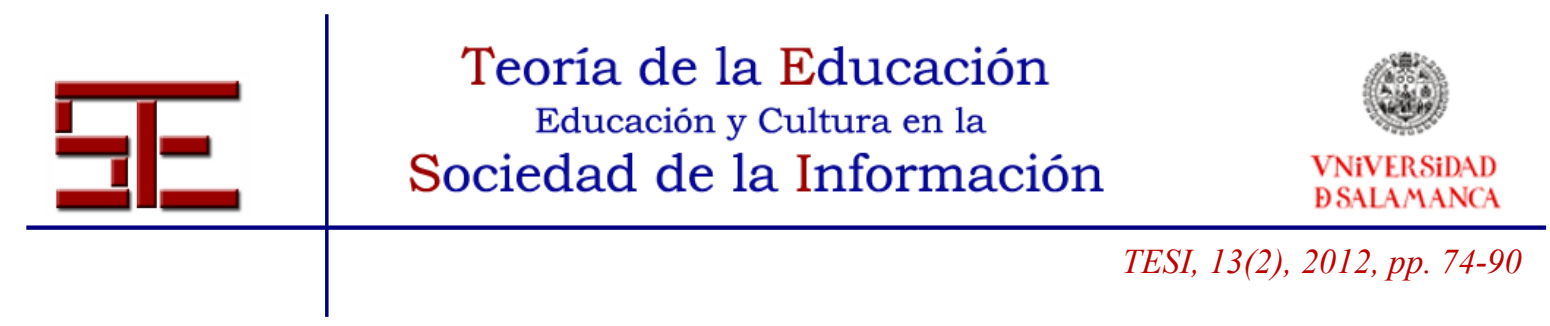

\title{
ROBOTIC EDUCATION, A TOOL FOR THE TEACHING-LEARNING OF THE SCIENCE AND TECHNOLOGY
}

\begin{abstract}
This paper presents and analyzes the educational robotics as a tool to support teaching and learning process at the level of pre-media, primarily engaged in complex subjects such as mathematics, physics and computer science, among others. The study was limited to high schools in the province of Chiriqui, Panama, took a sample of six schools in the province and for each school involved both students and teachers. The main objective of the project was to demonstrate how robotics education, facilitates and encourages teaching and learning of science and technology. The results showed that robotics could become an excellent tool to understand abstract concepts and complex subjects in the area of science and technology, as well as allowing developing basic skills such as teamwork.
\end{abstract}

Keywords: robotic education; teaching-learning process; skills; robotic kits.

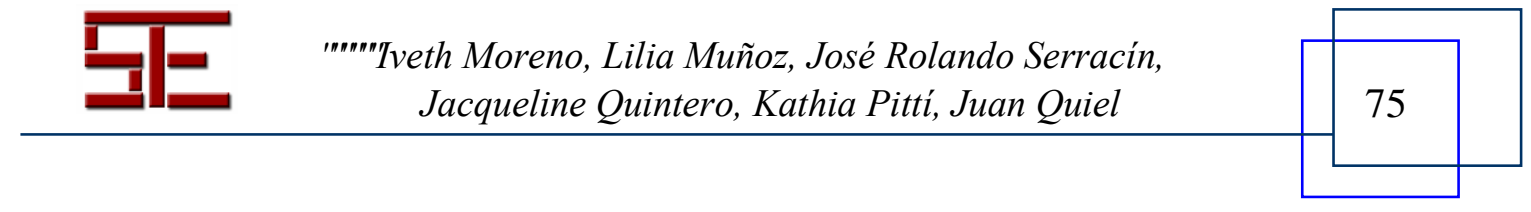




\section{LA ROBÓTICA EDUCATIVA, UNA HERRAMIENTA PARA LA ENSEÑANZA-APRENDIZAJE DE LAS CIENCIAS Y LAS TECNOLOGÍAS}

Fecha de recepción: 31/05/2012; fecha de aceptación: 18/06/2012; fecha de publicación: 25/07/2012

Iveth Moreno

Iveth.moreno@utp.ac.pa

Universidad Tecnológica de Panamá

Lilia Muñoz

lilia.munoz@rtp.ac.pa

Universidad Tecnológica de Panamá

José Rolando Serracín

jose.senacin@1tp.ac.pa

Universidad Tecnológica de Panamá

Jacqueline Quintero

jacqueline.quintero@;tp.ac.pa

Universidad Tecnológica de Panamá

Kathia Pittí Patiño

kathia_pitti@usal.es

Universidad de Salamanca

Juan Quiel

juanqh1508@hotmail.com

Universidad Tecnológica de Panamá

\section{1.- INTRODUCCIÓN}

Las empresas e industrias han incorporado procesos de producción y múltiples elementos tecnológicos que incluyen automatismos y control de procesos. Los ingenieros mecánicos, electrónicos y más recientemente los informáticos han asumido un papel protagónico en estos desarrollos. Sin embargo, también existen las demandas de las poblaciones jóvenes de contar con opciones de formación en esta especialidad (Zúñiga, 2006).

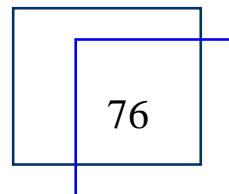

Iveth Moreno, Lilia Muñoz, José Rolando Serracín, Jacqueline Quintero, Kathia Pittí, Juan Quiel 


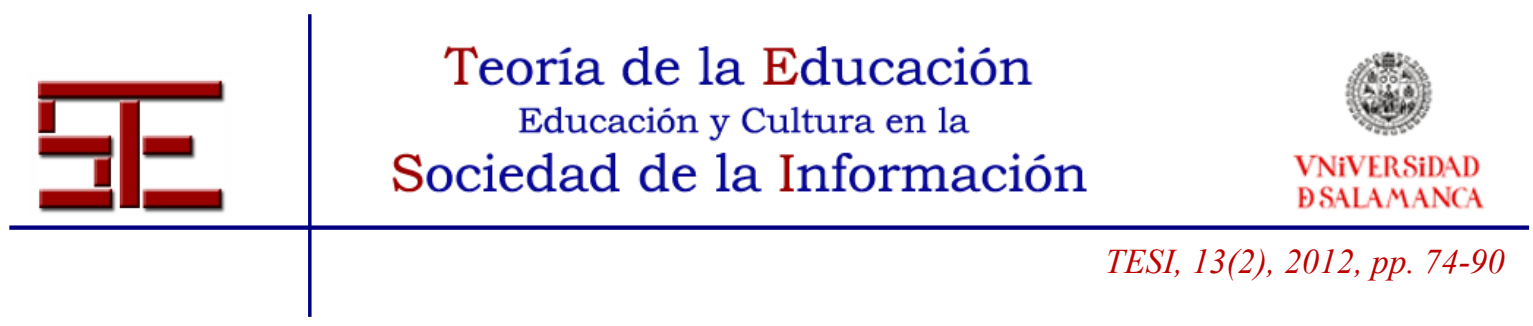

La robótica educativa se posiciona como un elemento nuevo y necesario de conocer por las nuevas generaciones. Utilizar la robótica en la educación implica el diseño y construcción de un robot. Siendo un robot un mecanismo controlado por un ordenador, programado para moverse, manipular objetos, hacer diferentes y determinados trabajos por medio de la interacción con su entorno (Encarta, 2008). La robótica educativa abarca temas multidisciplinarios como lo son: la electrónica, la informática, la mecánica y la física, entre otros.

Este artículo se desarrolla dentro del marco del Proyecto de Robótica Educativa APR- I-P09, financiado por la Secretaría Nacional de Ciencia, Tecnología e Innovación de la República de Panamá, y ejecutado por un grupo de docentes/investigadores de la Universidad Tecnológica de Panamá, Centro Regional de Chiriquí; y una doctoranda de la Universidad de Salamanca, España. El mismo estará estructurado de la siguiente manera: en la sección I, la introducción al artículo con el tema de la robótica educativa; en la sección II, la Metodología y Desarrollo utilizado en el proyecto; en la sección III los Análisis de los Resultados obtenidos durante el desarrollo del proyecto; en la sección IV se presenta las conclusiones y los trabajos futuros que se proponen a partir del proyecto; y por último en la sección $\mathrm{V}$ se presenta la bibliografía a la cual se hace referencia durante todo el artículo.

\section{1.- La Robótica Educativa}

La robótica educativa ha crecido muy rápidamente en la última década en casi todos los países y su importancia sigue aumentando. Esto parece ser un proceso lógico, ya que los robots están incorporándose en nuestra vida cotidiana, pasando de la industria a los hogares. Pero el propósito de utilizar la robótica en la educación, a diferentes niveles de enseñanza, va más allá de adquirir conocimiento en el campo de la robótica. Lo que se pretende es trabajar en el alumno competencias básicas que son necesarias en la sociedad de hoy día, como son: el aprendizaje colaborativo, la toma de decisión en equipo, entre otras (Educativa, 2011).

La robótica educativa es propicia para apoyar habilidades productivas, creativas, digitales y comunicativas; y se convierte en un motor para la innovación cuando produce cambios en las personas, en las ideas y actitudes, en las relaciones, modos de actuar y pensar de los estudiantes y educadores (Pozo, 2005). Si esos cambios son visibles en la práctica cotidiana, entonces estamos ante una innovación porque la

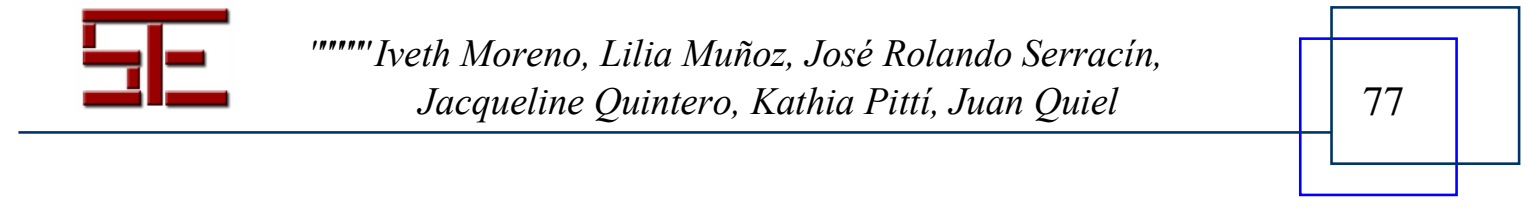




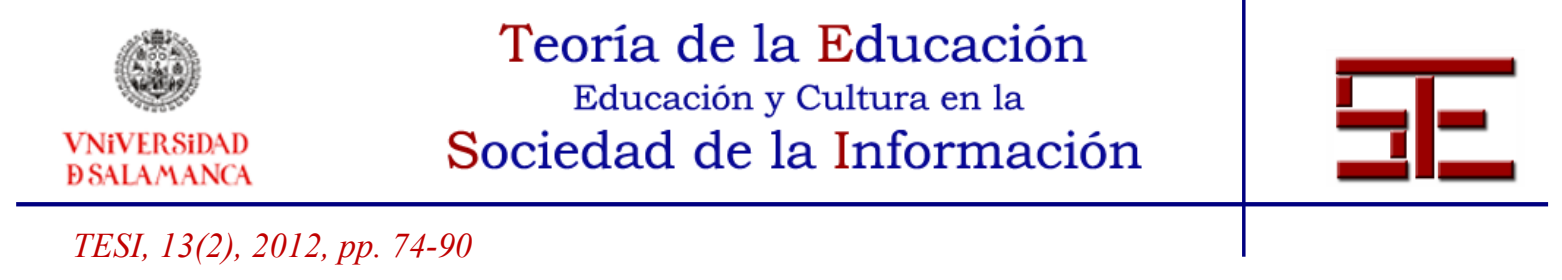

robótica habrá transcendido sus intuiciones y se reflejará en sus acciones y producto (Zúñiga, 2006).

La idea de implementar la robótica como apoyo a la educación tiene sus orígenes desde hace años, en 1983 el Laboratorio del Instituto Tecnológico de Massachusetts desarrolló el primer leguaje de programación educativo para niño llamado logos (Pozo, 2005). El surgimiento de kits de robótica ha ayudado a su inserción, ya que éstos se caracterizan por no exigir un conocimiento avanzado de electrónica o de programación.

Países como Corea e India empiezan a incluir la robótica en actividades fuera de clases, al ver los resultados que traía consigo, se reformó el esquema educativo con el cual se incluía la robótica dentro del aula (García, 2010).

La robótica en la educación se ha venido practicando en diferentes países de Asia, Europa, América y África como mencionan (García, 2010), (Mendoza, 2010), (Monsalves, 2011) y (Moreno et. al, 2011) entre otros; haciendo cada vez más popular el uso de la robótica educativa dentro y fuera de los planes curriculares de diferentes colegios secundarios y escuelas primarias alrededor del mundo.

La robótica educativa busca despertar el interés de los estudiantes transformando las asignaturas tradicionales (Matemáticas, Física, Informática) en más atractivas e integradoras, al crear entornos de aprendizaje propicios que recreen los problemas del ambiente que los rodea (Zúñiga, 2006). De esta manera hace frente a la crisis actual en la educación científica y que se debe principalmente a los métodos actúales de enseñanza que hacen a estas asignaturas difíciles y poco interesantes; sembrando en el estudiante una actitud negativa hacia la ciencia y tecnología, alejándolo de carreras y profesiones relacionadas con la ciencia.

Hoy en día la robótica se ha integrado en algunos programas de las escuelas primarias y secundarias, e incluso en los jardines de infancia. Esto se debe en parte a que la robótica provoca un alto nivel de atracción para los niños y jóvenes, muchas actividades educativas - cursos de robótica o competiciones de robots- dependen de esta fascinación por los robots móviles. El kit LEGO Mindstorms $\mathrm{NXT}^{1}$ es la plataforma más conocida para los estudios robóticos en etapas tempranas. Gallego (Gallego, 2010), reivindica la robótica educativa como vía para que los alumnos adquieran destrezas y

\footnotetext{
${ }^{1}$ LEGO Mindstorms NXT, http//:mindstorms.lego.com
}

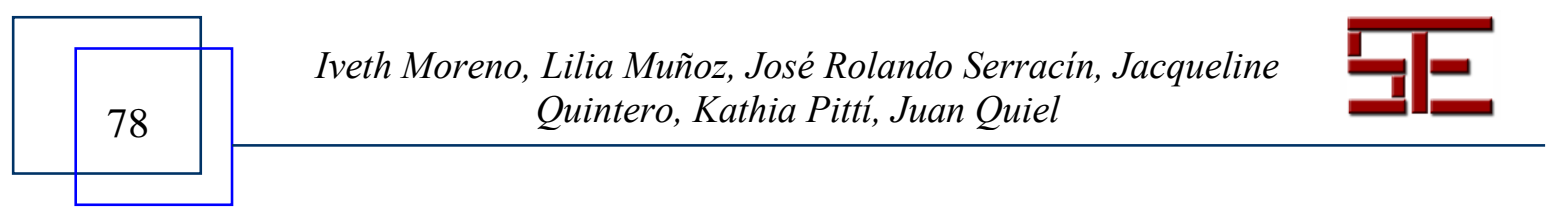




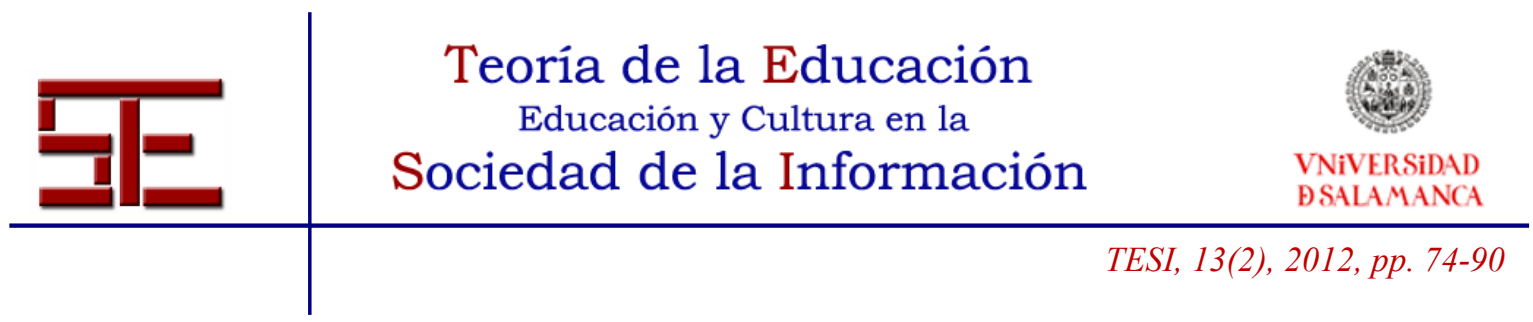

habilidades tecnológicas, pero también en el desempeño del trabajo en equipo (habilidades sociales).

\section{2.- Utilidad de la robótica en el proceso de enseñanza-aprendizaje}

El trabajo en equipo y la colaboración son la piedra angular de cualquier proyecto de robótica (Pittí, 2011). Al diseñar, construir y programar robots, los estudiantes pueden experimentar ese trabajar con la tecnología de una manera creativa e interesante, pero esto no es un proceso trivial, estas actividades, en un ambiente de enseñanzaaprendizaje adecuadamente diseñado, les ayudan a adquirir conocimientos de física, matemática, tecnología, programación, etc. Además, la resolución de problemas en equipos de trabajo colaborativo es un instrumento ideal para entrenar las competencias y habilidades blandas que son esenciales para hacer frente a estos procesos de desarrollo técnico y en el día a día (Pisciotta, 2010; Gallego, 2010; Ruiz-Velasco, 2007).

Uno de los objetivos de utilizar la robótica en las aulas es introducir a los estudiantes en las ciencias y la tecnología. Siguiendo el paradigma constructivista/construccionista y el aprendizaje a través del juego se puede contribuir a la construcción de nuevos conocimientos (Atmatzidou, 2008; Arlegui, 2008; Pittí, 2010; Savage, 2003). Por otra parte, las competiciones con robots son muy populares, ya que un desafío ofrece motivación extrínseca adicional para los estudiantes, aumenta sus habilidades de trabajo en equipo y anima al estudiante a identificar y evaluar una variedad de opiniones (Pisciotta, 2010).

Por consiguiente, existen diversos enfoques a la hora de enseñar a través de la robótica, todo dependerá de la manera en que se utilice durante el proceso de enseñanza-aprendizaje (Olaskoaga, 2009), que puede ser: como objeto de aprendizaje, como medio de aprendizaje o como apoyo al aprendizaje.

Los dos primeros enfoques implican que los contenidos se centren en la construcción y programación de robots, mientras que el tercer enfoque es el más importante pero menos conocido y desarrollado, donde los robots son utilizados en el aula como herramienta que favorece el acercamiento de un modo diferente a los contenidos del currículo, y que por sus propias características facilitan el aprendizaje por indagación.

Las relaciones aprendidas son simples pero importantes. El conocimiento puede ser de la física: la vibración (oscilación) frente a la rotación, transformación de la energía;

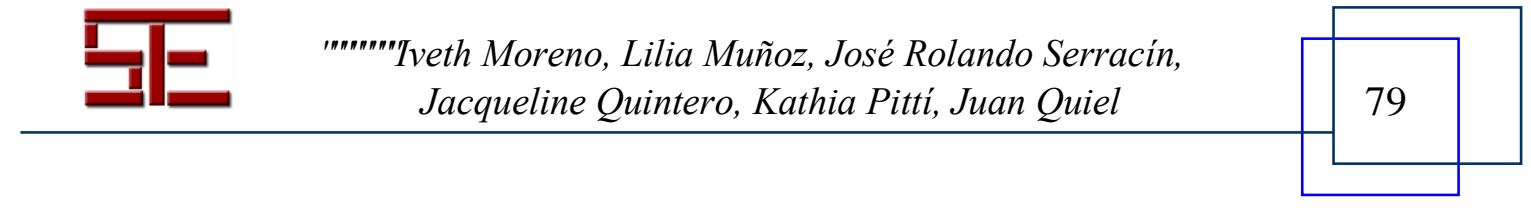




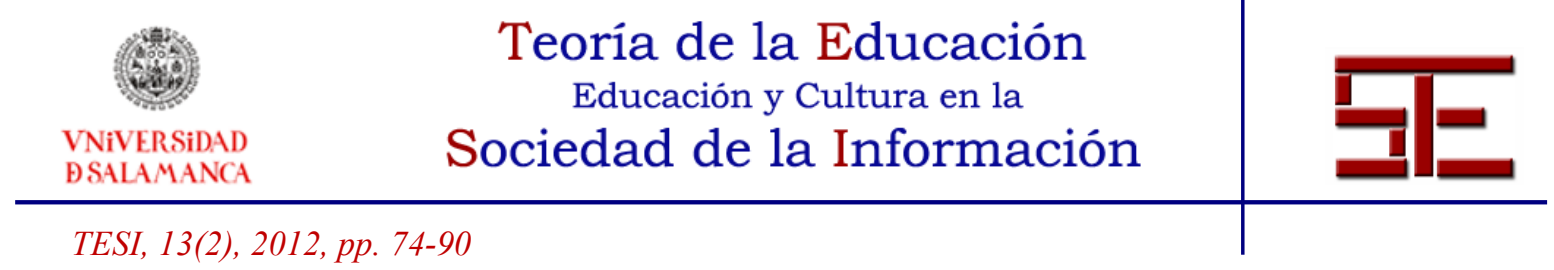

de la biología: sensores vs sentidos, el pensamiento frente a los programas, músculos vs mecanismo; de manera similar en las matemáticas, lenguaje, tecnología, artes, y el mundo que les rodea. El aprendizaje se hace más rico y el entendimiento más profundo, al poder trasladar el conocimiento de un objeto y verlo en otro contexto.

Para Gallego (Gallego, 2010), la importancia de la robótica educativa radica en que:

- Aglutina ciencias y tecnologías: matemáticas, física, informática...

- Fomenta la imaginación, despierta inquietudes y ayuda a comprender mejor el mundo que nos rodea.

- Permite el trabajo en equipo facilitando la comunicación, responsabilidad, toma de decisiones...

Otro aspecto que se debe destacar es que los estudiantes aprenden que es aceptable cometer errores, especialmente si esto les lleva a encontrar mejores soluciones. Se aprende más de un error que de un acierto. Nos ayuda a intentar superarnos. Si todo funciona bien y rápido no aporta demasiado.

\section{2.- METODOLOGÍA DESARROLLADA}

En el caso de Panamá, la robótica educativa se ha venido desarrollando como una actividad extracurricular en colegios secundarios de la ciudad capital del país, participando en pequeñas competencias amistosas. Hasta el momento del desarrollo del proyecto, en la Provincia de Chiriquí no existía Colegio Secundario o Escuela Primaria que hubiera experimentado con la robótica educativa en sus aulas de clase.

El Proyecto de Robótica Educativa que se describe tuvo como objetivo general demostrar como la robótica aplicada a la educación facilita y motiva la enseñanzaaprendizaje de las ciencias y las tecnologías, como asignatura multidisciplinaria; dirigida tanto a estudiantes como a docentes de los colegios secundarios, utilizando los Mindstorms NXT de Lego, como elemento robótico de bajo costo. Para ello, el proyecto se desarrolló en tres etapas. La primera consistió en la revisión bibliográfica, giras de entrenamiento, formación de los grupos participantes, lanzamiento formal del proyecto, preparación de ayudantes-instructores, diseño y desarrollo de un sitio Web. La segunda consistió en el desarrollo propio de los cursos con la participación de estudiantes y docentes de seis colegios diferentes que involucraron a tres distritos diferentes de la provincia y en esta etapa se desarrolló una serie de contenidos que formarían a los participantes en el uso de los Mindstorms NXT de Lego. En la tercera etapa los

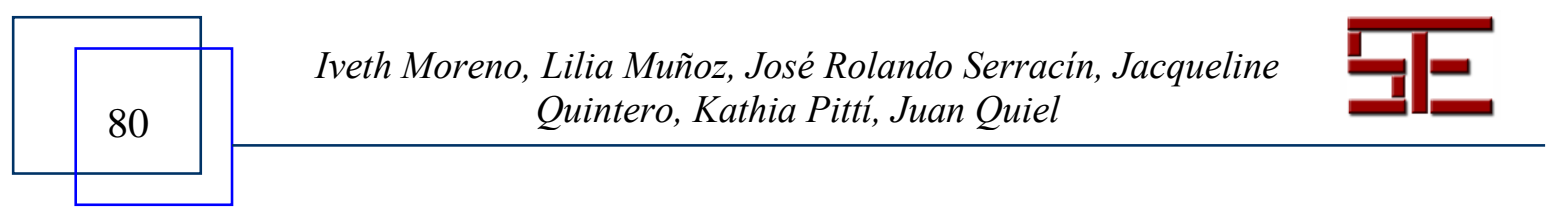




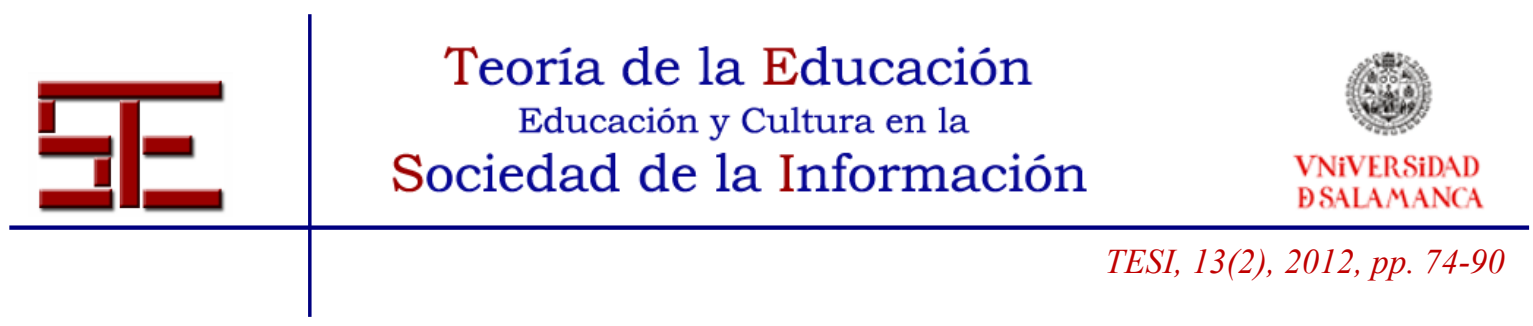

estudiantes y docentes participaron de una videoconferencia con la Universidad de Salamanca, pusieron en práctica y de una forma autónoma nuevas aplicaciones y formaron grupos para participar en competencias amistosas con los demás compañeros.

En la primera etapa, se entrenó a los ayudantes del proyecto, se participó en reuniones de cooperación con el Centro de Tecnologías Avanzadas, CITA, de la Fundación Germán Sánchez Ruipérez, en Salamanca España, y se adquirió el equipo que se utilizaría para la implementación del proyecto. Además, en esta etapa, cada colegio eligió los docentes y estudiantes que participarían en el proyecto. Se les solicitó previamente a los directores de los colegios, que eligieran a tres docentes de las áreas científicas o tecnológicas, y que además por lo menos uno de ellos les dictará clases a los estudiantes que participarían del Proyecto. En el caso de los estudiantes, los colegios tuvieron criterios propios de selección, tales como los mejores estudiantes, o los que querían participar, o los que presentaban una mejor conducta en el aula de clase.

Durante la segunda etapa, se formaron tres grupos, dos grupos de estudiantes y un grupo de profesores. En cada grupo se tuvieron 18 participantes, seis kits de la Lego Education y seis ordenadores; por lo tanto, tres participantes utilizaron un kit y un ordenador para realizar los programas informáticos que se instalaron en los diferentes modelos robóticos implementados por los grupos. Los participantes fueron entrenados en temas de robótica, tales como diseño mecánico, electrónica y programación; durante 4 horas, una vez a la semana, por 12 semanas. Cabe mencionar que en cada grupo existía un programador, un ingeniero y un periodista; y estas tres funciones se rotaban dentro del grupo de tres participantes en cada sesión. Esto último permitió a cada uno de los participantes experimentar los tres roles, reforzar fortalezas y superar debilidades.

En la Figura 1 se observa tanto a los docentes como a los estudiantes realizando actividades que corresponden a la segunda etapa del proyecto, en donde se formaba a los participantes en el uso de la robótica como herramienta que se puede utilizar en el proceso educativo para las asignaturas de las áreas científicas-tecnológicas.

Por otro lado, de los seis colegios participantes, dos colegios pertenecían a comunidades rurales y los otros cuatro a comunidades urbanas. Entre los estudiantes, 24 eran del sexo femenino y 12 del sexo masculino; es más, en un colegio todo el equipo estaba conformado por niñas. Por el lado de los docentes, se contó con la participación de 10 docentes del sexo masculino y 8 docentes del sexo femenino, que dictaban indistintamente asignaturas de matemáticas, informática, física y química.

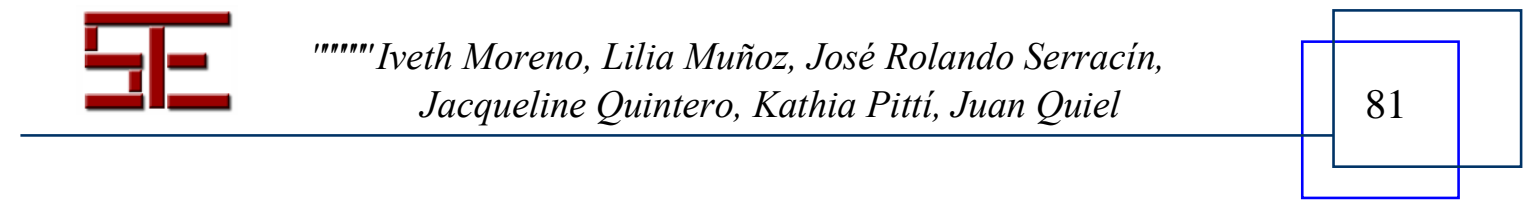




\begin{tabular}{cc} 
Teoria de la Educación \\
Educación y Cultura en la \\
$\begin{array}{c}\text { VNiVERSIDAD } \\
\text { DSALAMANCA }\end{array}$ \\
\hline TESI, 13(2), 2012, pp. $74-90$
\end{tabular}
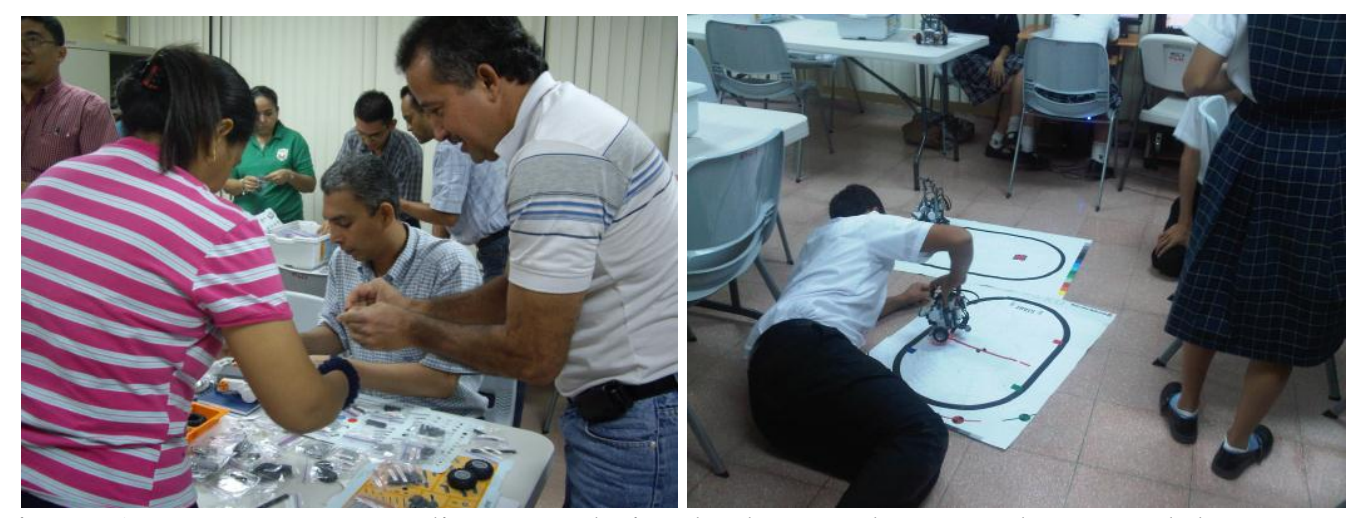

Figura 1. Docentes y estudiantes trabajando durante la segunda etapa del proyecto.

La tercera etapa fue productiva e interesante. Los profesores participantes llevaron a sus colegios la robótica educativa aplicándola a sus clases de matemáticas, física, química y de informática. Luego, tanto profesores como estudiantes participantes del proyecto teleoperaron un robot situado en Salamanca y compartieron experiencias aprendidas con los miembros del grupo de robótica educativa de CITA. El proyecto finalizó con la Primera Competencia Amistosa Intercolegial de Robótica, celebrada en la Universidad Tecnológica de Panamá, Centro Regional de Chiriquí; donde se realizaron pruebas de laberintos, misiones, competencia de sumo y presentación de proyectos. En la Figura 2 se pueden observar fotos del día de la competencia, en la categoría de lucha de sumo; y del día de la videoconferencia con el Centro de Tecnologías Avanzadas (CITA), de la Fundación Germán Sánchez Ruipérez; de izquierda a derecha respectivamente.
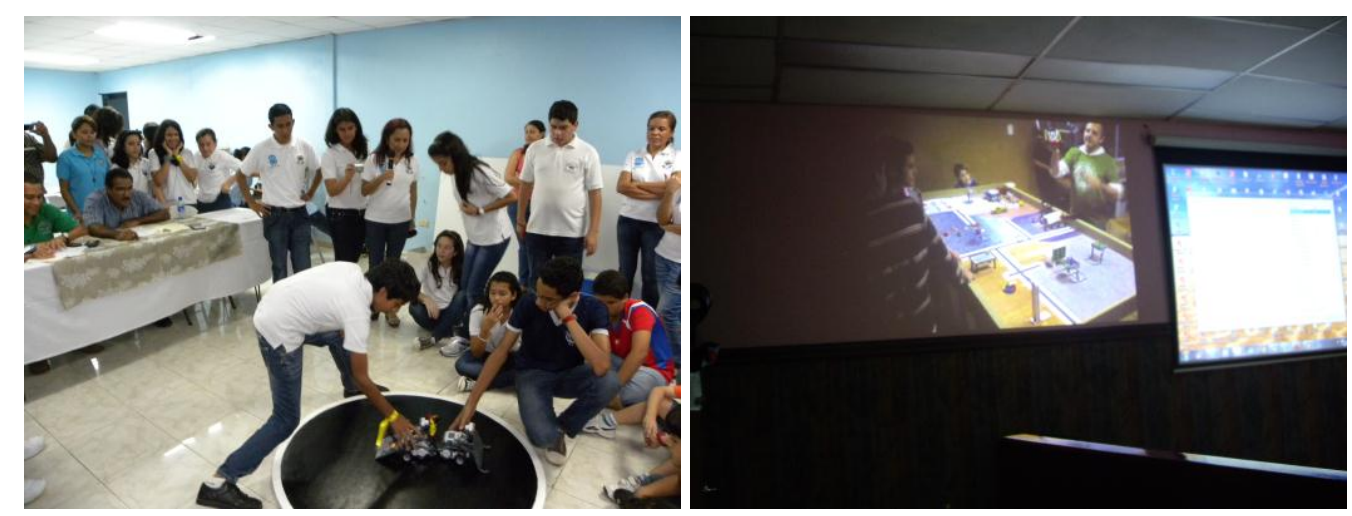

Figura 2. Actividades realizadas durante la tercera etapa del proyecto.

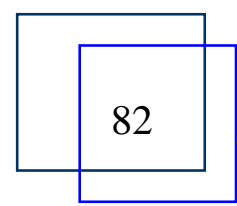

Iveth Moreno, Lilia Muñoz, José Rolando Serracín, Jacqueline Quintero, Kathia Pittí, Juan Quiel 


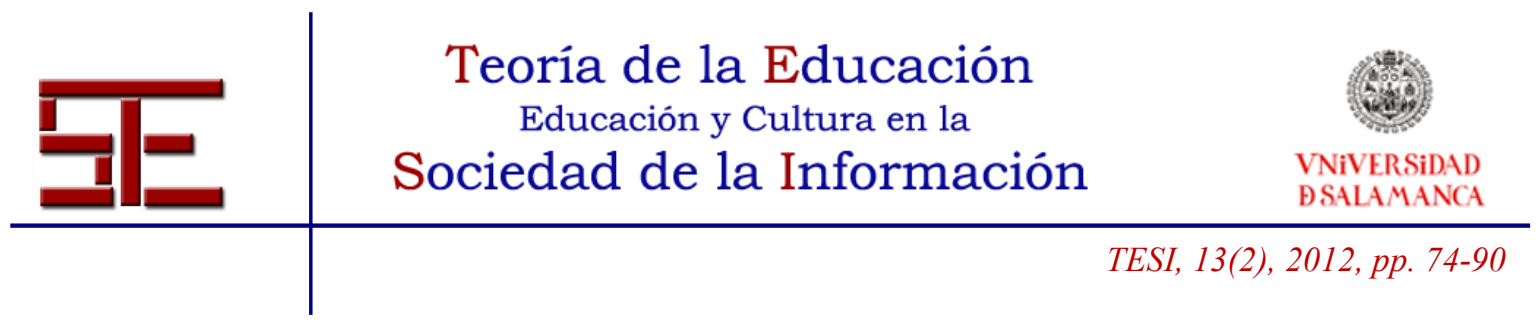

\section{3.- ANÁLISIS DE RESULTADOS}

\section{1.- Evaluación a docentes.}

La evolución de los docentes fue evaluada mediante una encuesta según la escala ReNXT, donde cada docente realizaba una autoevaluación. Las preguntas realizadas estaban enfocadas en descubrir cómo consideraban sus conocimientos en robótica, mecánica, matemáticas, ciencias y otras áreas del saber; qué tan capaces se sentían en expresar y transmitir sus conocimientos; y por último se trataba de medir su capacidad para reconocer los sentimientos propios y ajenos, en otras palabras su habilidad emocional. Cabe señalar que se pudieron aplicar otros tipos de evaluaciones a los docentes, por ejemplo, una realizada por los capacitadores. Pero tanto en el caso de los docentes como de los estudiantes sólo se realizó la encuesta mencionada anteriormente.

La Tabla 1 muestra los gráficos que resumen los resultados de las encuestas. En cada uno de los tres grupos en que han dividido las habilidades del docente se ha observado un incremento en la cantidad de docentes que considera buena una habilidad particular. Llama la atención el hecho de que en lo que se refiere a inteligencia emocional, si bien antes de iniciar el curso ninguno de los participantes se consideraba malo, al finalizar el mismo casi un $1 \%$ sentía que tenía una deficiencia en sus relaciones interpersonales e intrapersonales, tal vez esto se deba a esta faceta, de trabajo en equipo, que debe desarrollarse en las sesiones prácticas y que tal vez algunos de ellos ya hace mucho no realizaban, por lo menos no de esta forma. Por otro lado, a pesar de que los docentes lo eran de materias como ciencias, física y matemática, algunos no se sentían preparados en la mecánica y la robótica; y esta experiencia ayudó a reforzar estas áreas, que son de provecho para aplicación de las ciencias. En cuanto a la tercera columna es obvio que algunos docentes sienten que la forma en que transmiten los conocimientos no es del todo buena, esto se entiende debido a los resultados que obtienen usualmente los estudiantes en sus materias; queremos creer que un aumento en el número de docentes que consideran que expresan sus conocimientos de forma verbal y escrita, de una buena manera, sea indicativo también de que los resultados de las evaluaciones a sus estudiantes sean mejores. En fin, tal vez se requiera un estudio más profundo de la influencia de la robótica educativa en el docente, sin embargo, en términos generales los resultados indican que existe una mejoría del docente en casi todas las áreas del conocimiento; y sobre todo consideran que el uso de la robótica como herramienta para la enseñanza de las ciencias y las tecnologías favorece notablemente el proceso educativo.

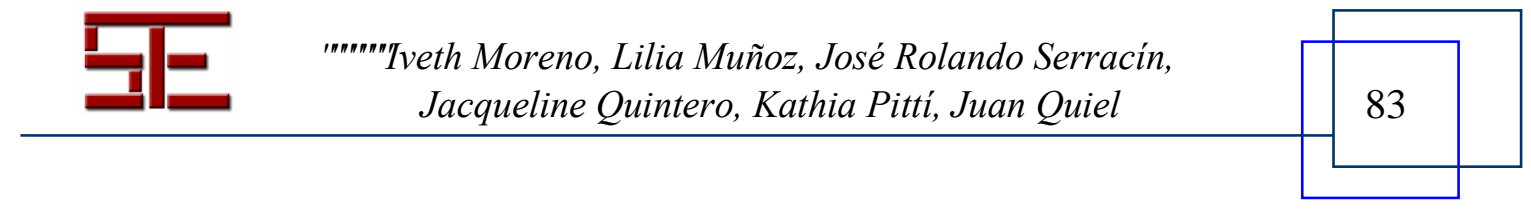




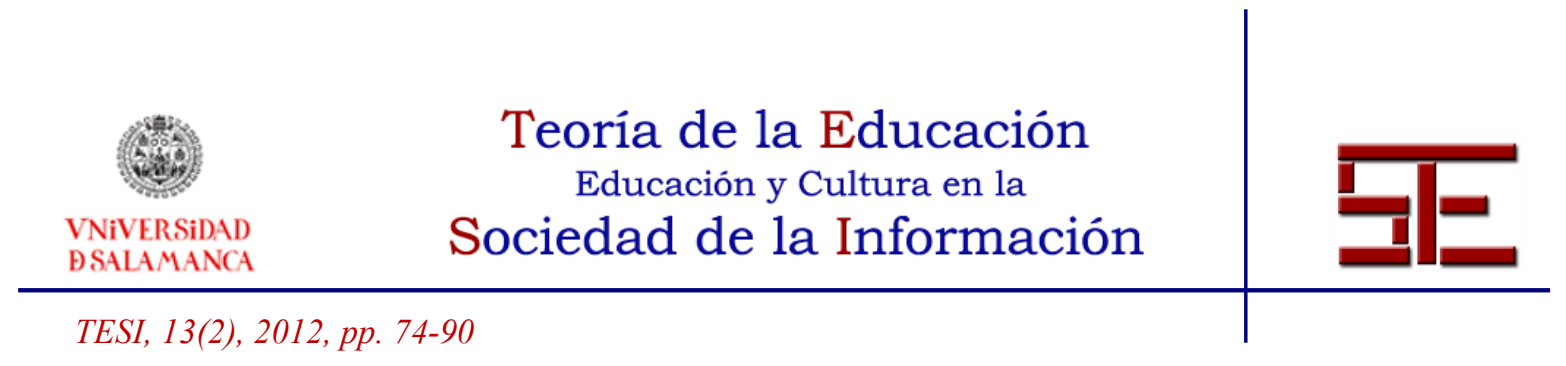

Tabla. 1

Resultados de las encuestas realizadas a los docentes

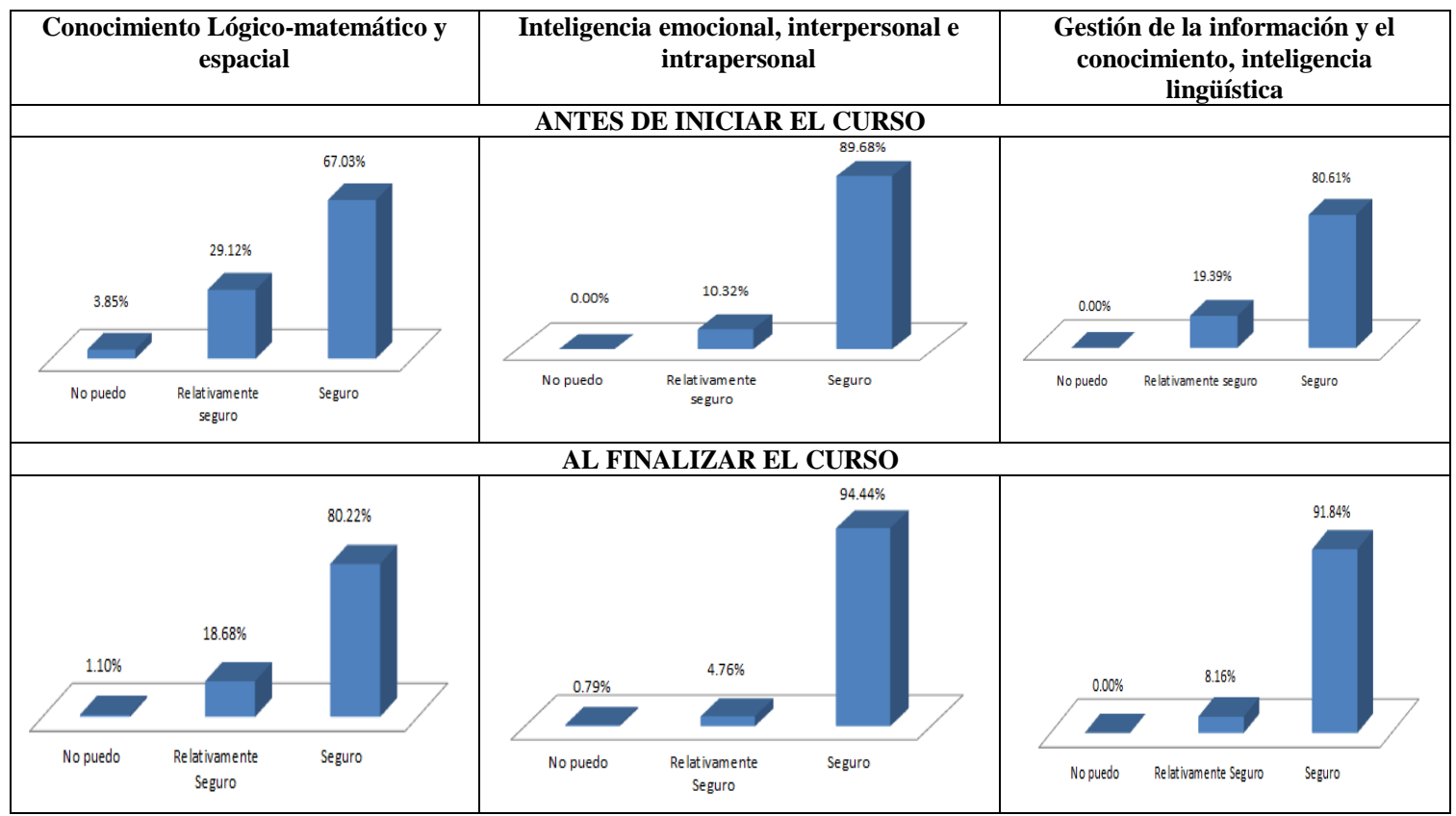

Por otro lado, en la Tabla. 2 se presentan algunos resultados de manera independiente, según las categorías mencionadas en la Tabla. 1. Todos los docentes presentaron una mejoría notable en cada uno de los aspectos analizados, y resulta interesante observar como la capacidad de hablar en público para los docentes cambió hasta un $100 \%$; un aspecto que es de gran ayuda al docente en el proceso de enseñanza.

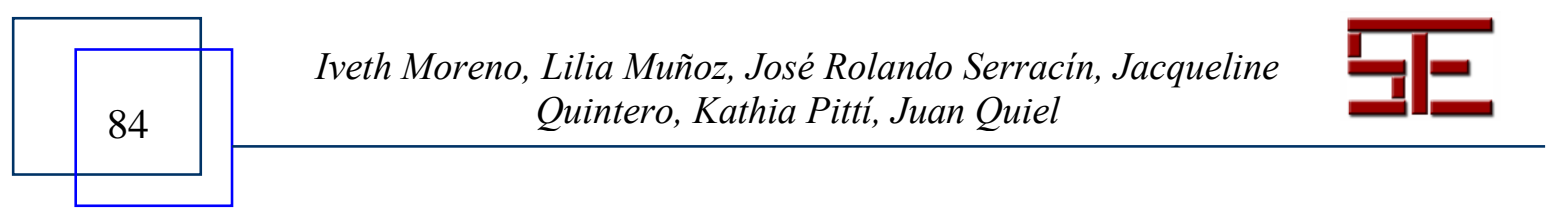




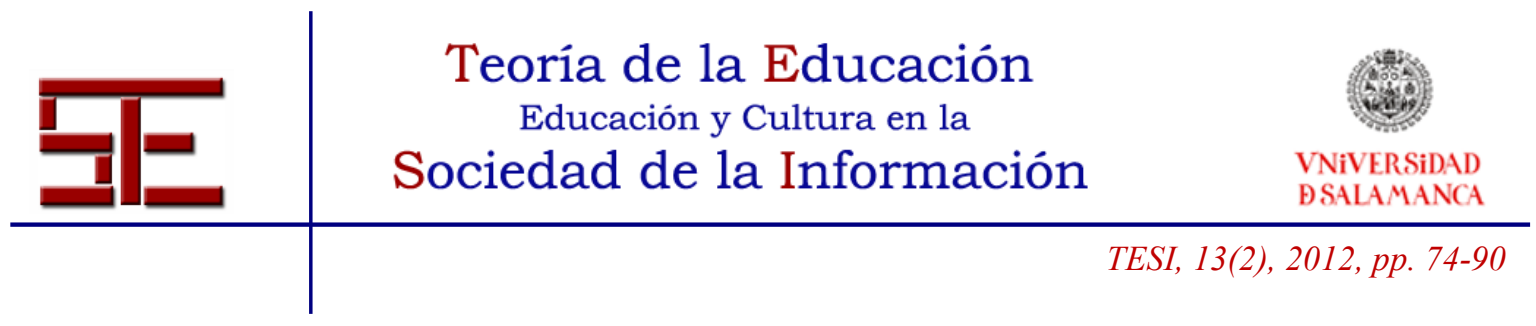

Tabla. 2

Resultados independientes de la encuesta realizada a los docentes

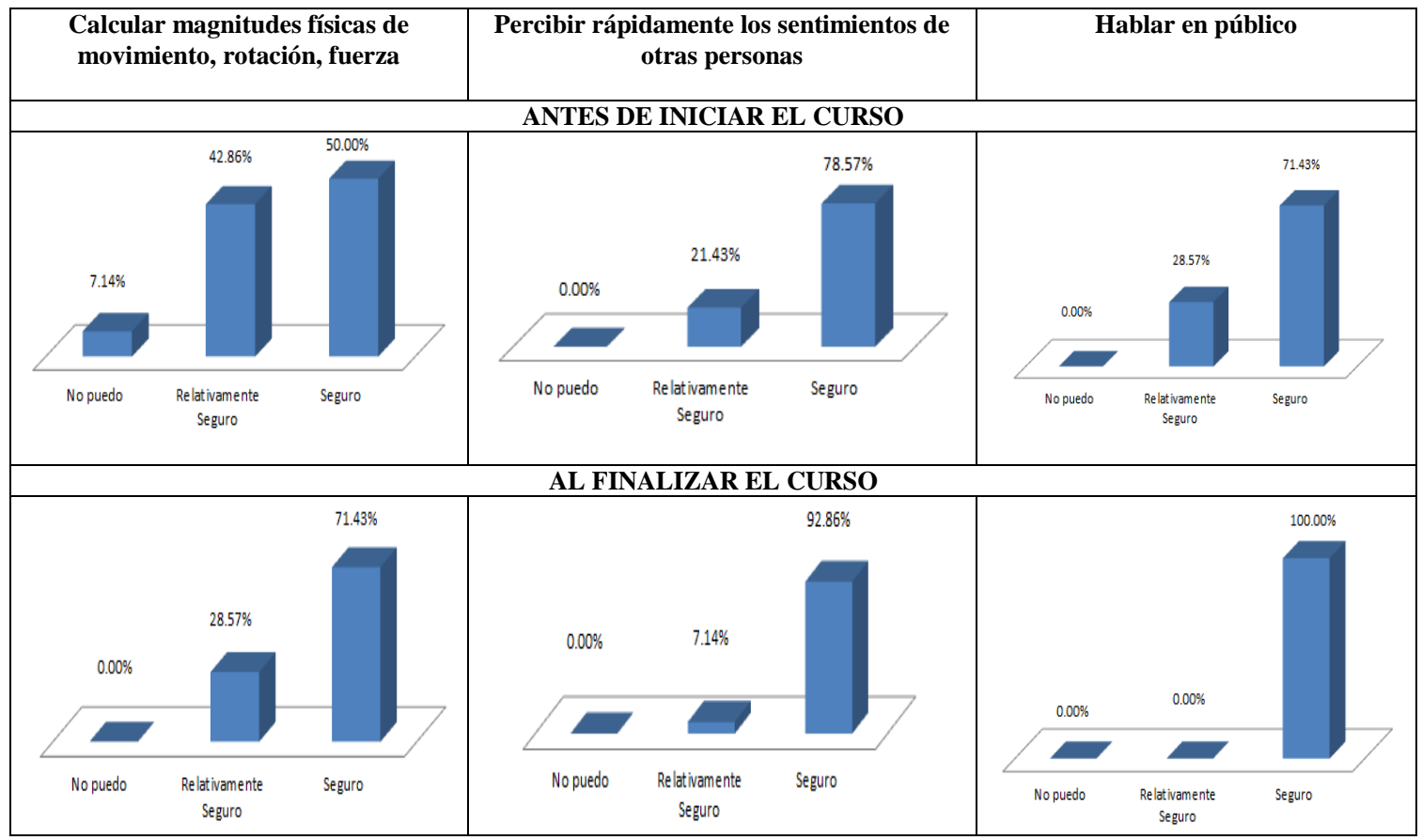

\section{2.- Evaluación a estudiantes}

$\mathrm{Al}$ igual que a los docentes a los estudiantes también se le aplicó una encuesta al inicio al finalizar el proyecto. En la Tabla 3 se muestran los gráficos resumen de las encuestas aplicadas a los estudiantes. En los grupos en que se dividieron las habilidades de los estudiantes se nota el crecimiento. En la primera columna principalmente se puede apreciar el aumento significativo en las destrezas lógicas y matemáticas, un aumento del $15.87 \%$ con respecto al inicio del curso. Por otro lado, la confianza en sí mismos aumentó al descubrir que tienen el poder de crear objetos (robots), lo que al inicio les parecía demasiado complejo. En la segunda columna se puede apreciar la información relativa a la inteligencia emocional e interpersonal, cabe mencionar que este aspecto se mantuvo casi igual al final del curso. En la tercera columna se puede observar el resultado obtenido en la gestión de la información, el logro de la concentración y la disciplina, y la colaboración a partir del trabajo en equipo, el aumento en este aspecto se

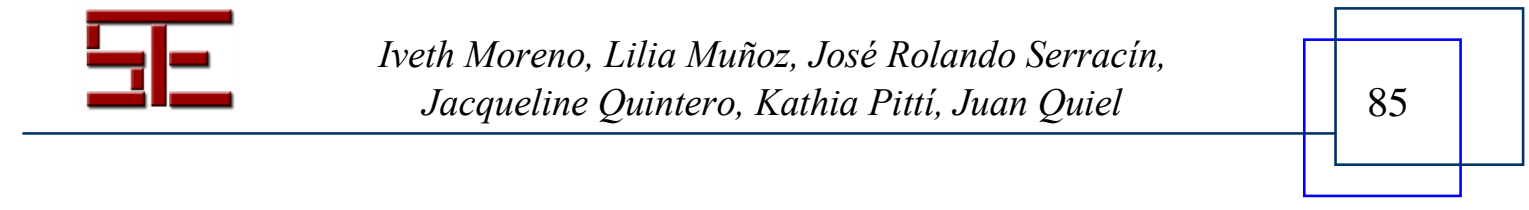




\begin{tabular}{c} 
Teoría de la Educación \\
Educación y Cultura en la \\
$\begin{array}{c}\text { VNiVERSIDAD } \\
\text { BSALAMANCA }\end{array}$ \\
\hline TESI, 13(2), 2012, pp. 74-90
\end{tabular}

dio en un $14.44 \%$. Es importante mencionar que en este proceso de capacitación la calidad de aprendizaje de los estudiantes ha aumentado, pues permanecieron motivados durante toda la capacitación, en parte por la construcción de robots y la posibilidad de controlar su funcionamiento a través de la programación.

Adicionalmente se consideró realizar una evaluación del grado de confianza en función del género considerando que en el curso participaron niñas y niños. En la Tabla 4 se pueden apreciar los resultados de esta evaluación, los cuales indican que al inicio los niños tenían mayor grado de confianza del $64.58 \%$ de estar seguros de poder realizar las tareas y este aumentó en un $19.51 \%$ al finalizar el curso, mientras que las niñas tenían un menor grado de confianza con solo un $28.13 \%$ al estar seguras de poder realizar las actividades presentadas, sin embargo es notable el aumento significativo obtenido por las niñas al finalizar el curso con un aumento del grado de confianza de un $33.77 \%$. De lo cual podemos deducir que el grado de confianza de las niñas con respecto a los varones aumentó significativamente.

Tabla. 3

Resultados de las encuestas realizadas a los estudiantes

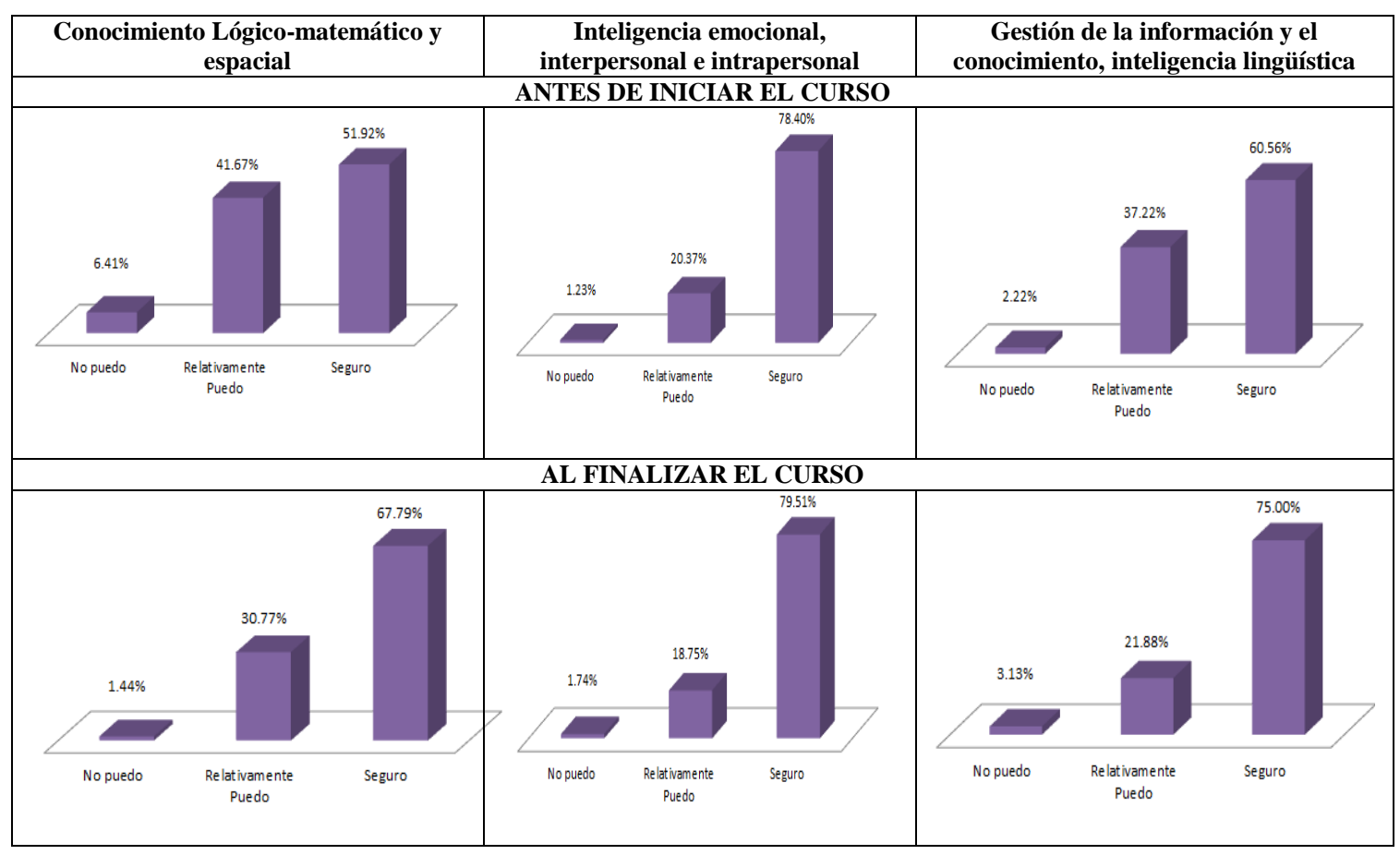

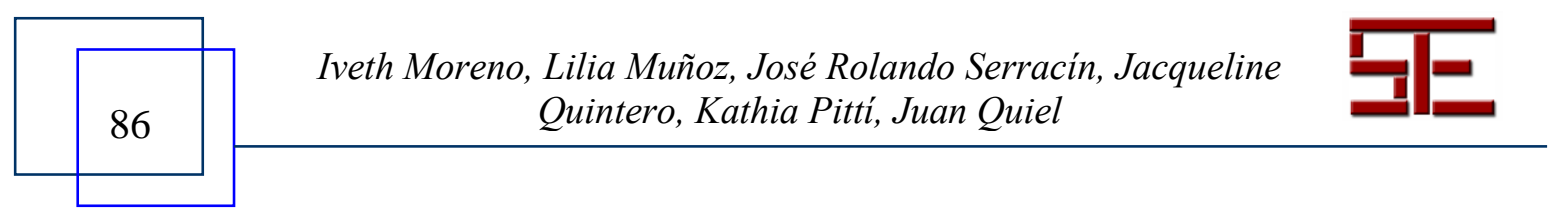




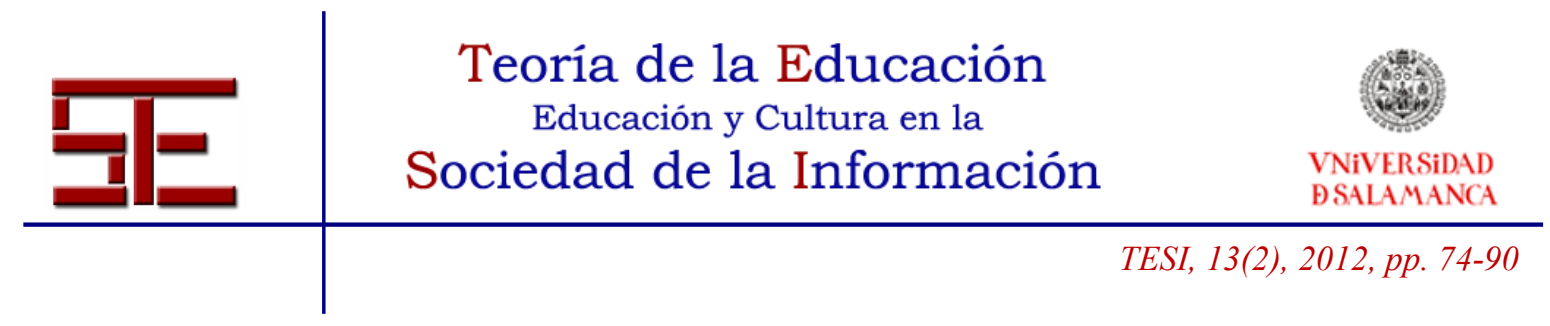

Tabla. 4

Resultados de las encuestas del grado de confianza por género

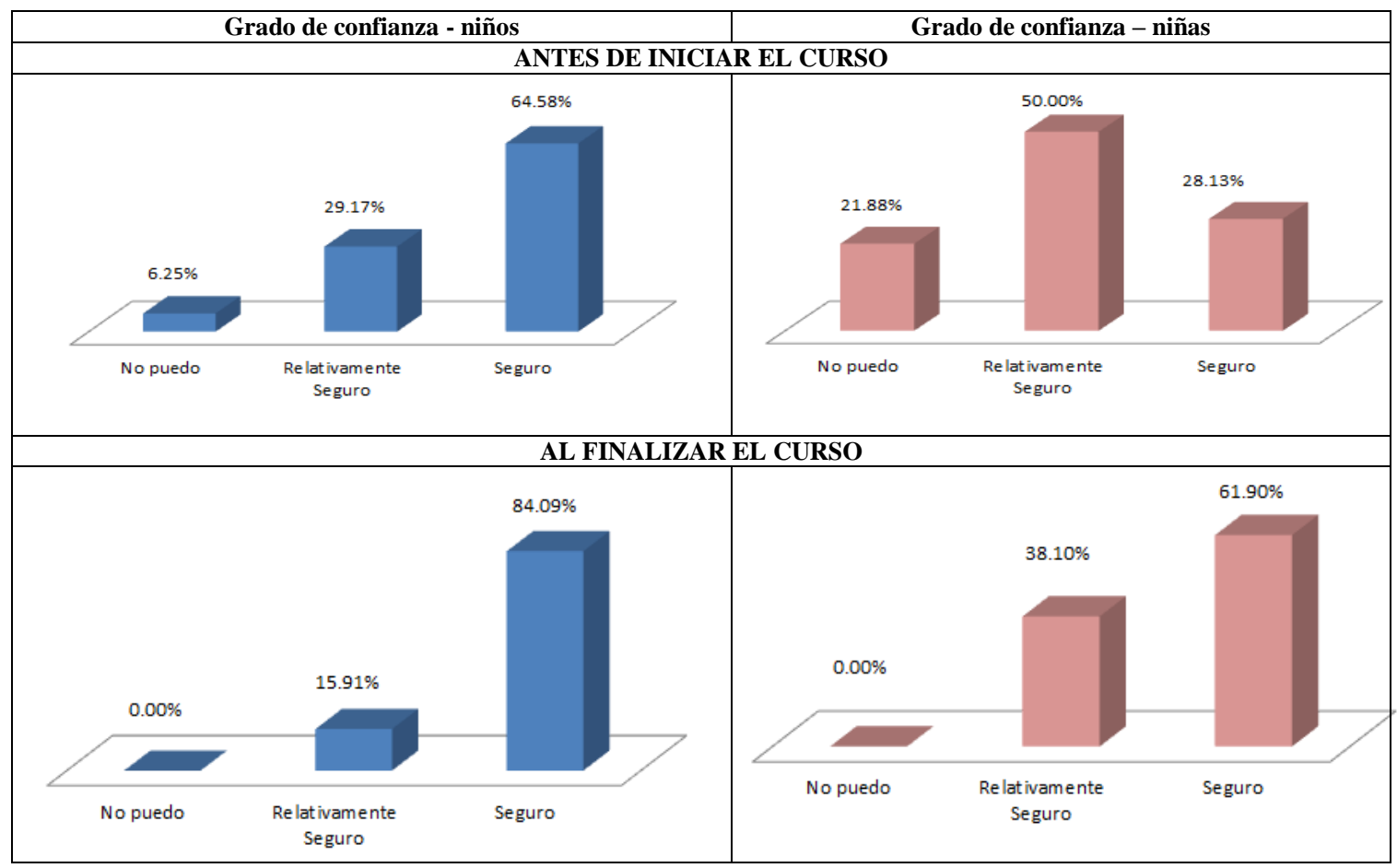

Otro aspecto interesante fueron los resultados que se observaron al evaluar a los estudiantes con respecto al tipo de comunidad. En la Tabla. 5 se puede observar con, respecto al aspecto "Conocimiento Lógico-matemático y espacial", que el aprovechamiento de los estudiantes de colegios de zonas rurales fue más significativo que el de los estudiantes de colegios de zonas urbanas.

\section{4.- CONCLUSIONES Y TRABAJOS FUTUROS}

La necesidad de contar con escenarios diferentes para lograr vencer las barreras en el proceso enseñanza-aprendizaje justifica el desarrollo del proyecto que presentamos en este artículo, siendo uno de los principales retos despertar el interés de estudiantes y docentes en el uso y la aplicación de la robótica educativa en el aula. Además, dota al

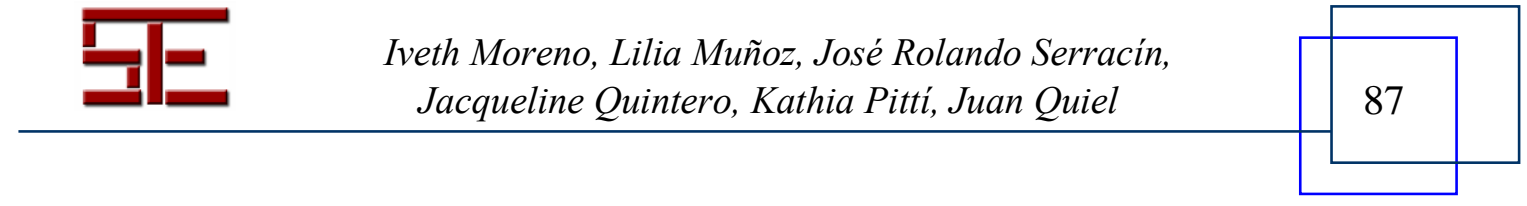




\begin{tabular}{c} 
Teoría de la Educación \\
Educación y Cultura en la \\
$\begin{array}{c}\text { VNiVERSIDAD } \\
\text { BSALAMANCA }\end{array}$ \\
\hline TESI, 13(2), 2012, pp. 74-90
\end{tabular}

estudiante de un espacio controlado en donde puede cometer errores y no generar perjuicio en el propio estudiante (se aprende más de los errores).

Con el desarrollo de este proyecto ha quedado demostrado que la utilización de la robótica educativa empleada como herramienta adicional para la docencia mejora la atención del estudiante y también la productividad del docente. En cuanto al docente, renueva su compromiso por estar actualizado y el grado de satisfacción del docente respecto al proceso enseñanza aprendizaje es mucho mayor. Por otro lado, desde el punto de vista de los estudiantes el proyecto ha permitido despertar el interés por la investigación y la búsqueda de soluciones que generen nuevo conocimiento.

Por su parte, el tema de la formación y capacitación del docente fue algo innovador en el proyecto, ya que por lo menos en Panamá sólo se había trabajado la robótica educativa con los estudiantes. Adicional, podemos mencionar que la robótica educativa nos ofrece un vasto campo para la investigación y un nuevo enfoque para la academia, que desarrolla y refuerza competencias necesarias para los futuros campos laborales.

Finalmente, como trabajo futuro se pretende extrapolar esta experiencia a estudiantes de otros niveles académicos y de otras regiones del país.

Tabla. 5

Resultados de las encuestas aplicadas a los estudiantes según el tipo de comunidad

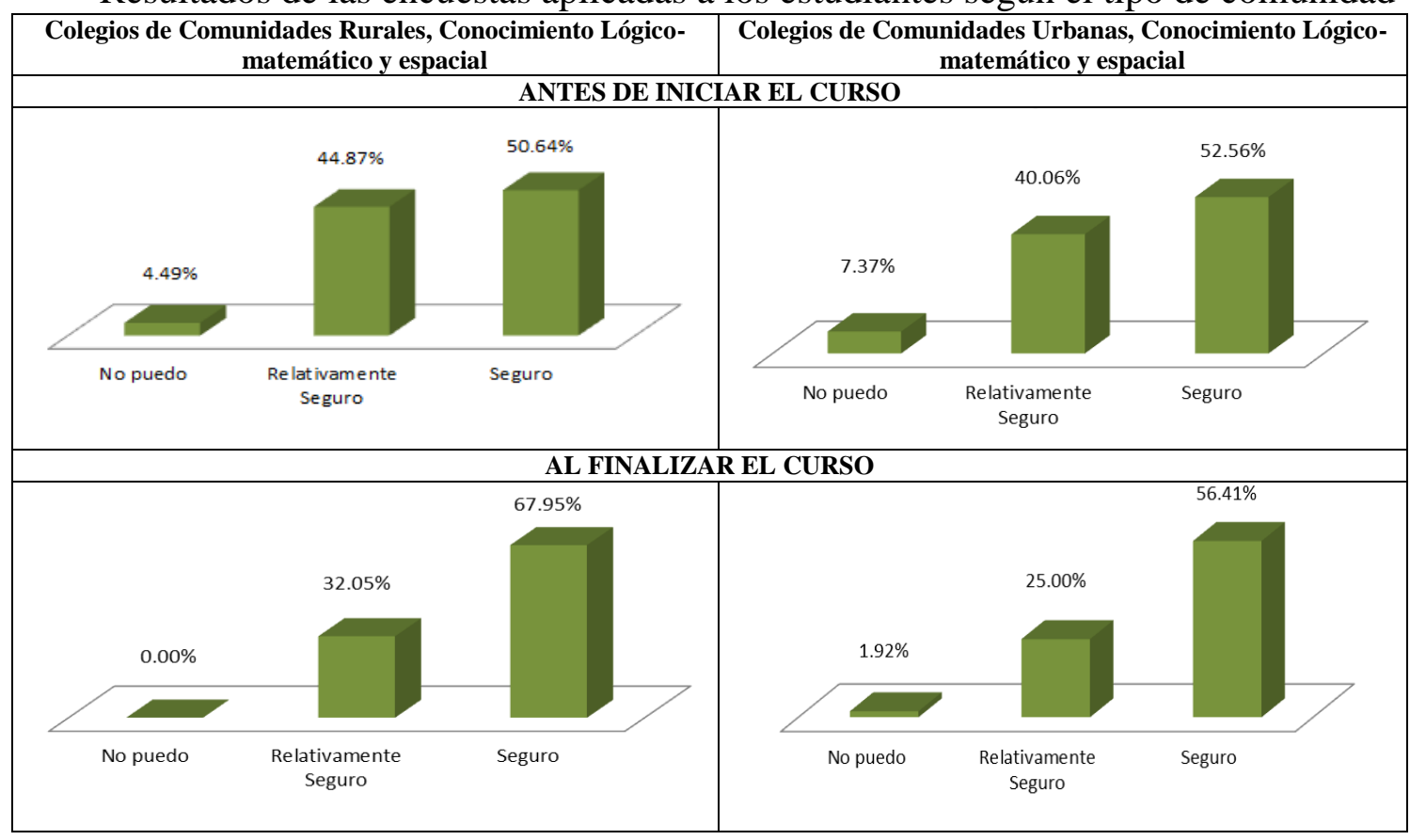

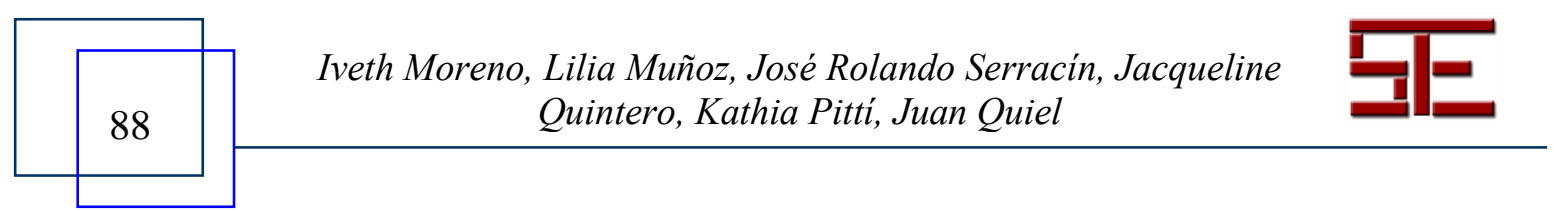




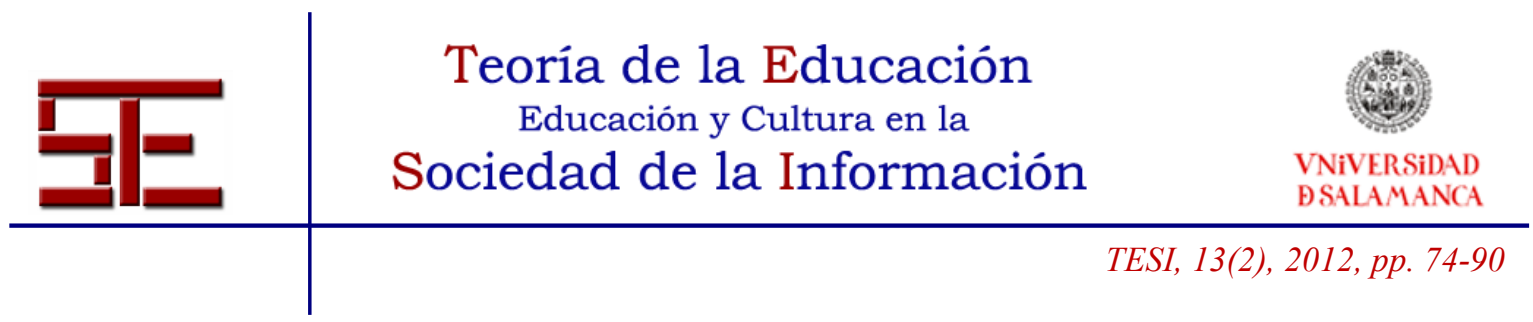

\section{5.- BIBLIOGRAFÍA}

Arlegui, J., Menegatti, E., Moro, M., Pina, A. (2008). Robotics, Computer Science curricula and Interdisciplinary activities. En Workshop Proceedings of SIMPAR 2008 Intl. Conf. On Simulation, Modeling and Programming for Autonomous Robots, pp. 1021.

Atmatzidou, S., Markelis, I., Dimitriadis, S. (2008). The use of LEGO Mindstorms in elementary and secondary education: game as a way of triggering learning. En Workshop Proceedings of SIMPAR 2008 Intl. Conf. on On Simulation, Modeling and Programming for Autonomous Robots, pp. 22-30.

Wikipedia (24 de mayo de 2011). Recuperado el 15 de junio de 2011, de http://es.wikipedia.org/wiki/Rob\%C3\%B3tica_educativa.

Educativa, D. d. (2011). Guía Didáctica para el responsable del Programa de Robótica Educativa. Sinaloa.

Enciclopedia Microsoft Encarta 2008.

Gallego, E. (2010). Robótica Educativa con Arduino una aproximación a la robótica bajo el hardware y software libre. Extraído el 18, de mayo, 2012, de http://anteriores.eventos.cenditel.gob.ve/site_media/detalle/files/robotica.pdf.

García, E. M. (agosto de 2010). Guía Didáctica para el Responsable del programa Robótica Educativa. Recuperado el 8 de mayo de 2011, de http://www.dtesepyc.gob.mx/archivos/guia_dicatica_robotica.pdf.

Mendoza P. (13 de abril de 2010). El e-espacio de los contenidos digitales de la UNED. Recuperado el 20 de mayo de 2011, de http://eespacio.uned.es/fez/view.php?pid=taee:congreso-2010-1033.

Monsalves, S. (2011). Estudio sobre la utilidad de la robótica educativa desde la perspectiva del docente. Revista de Pedagogía, 32 (90), 81-117.

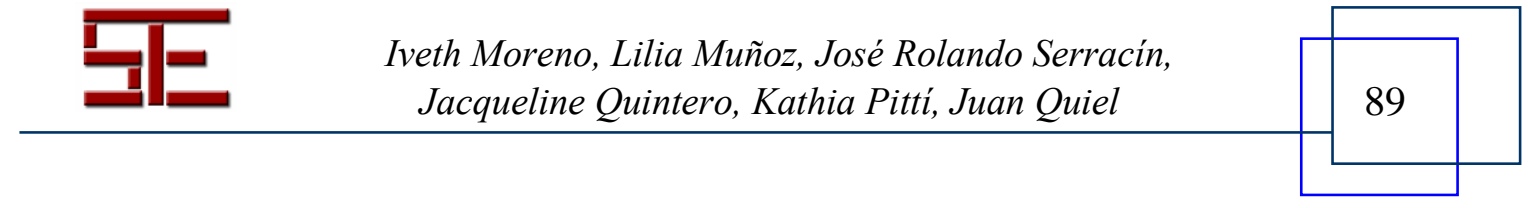




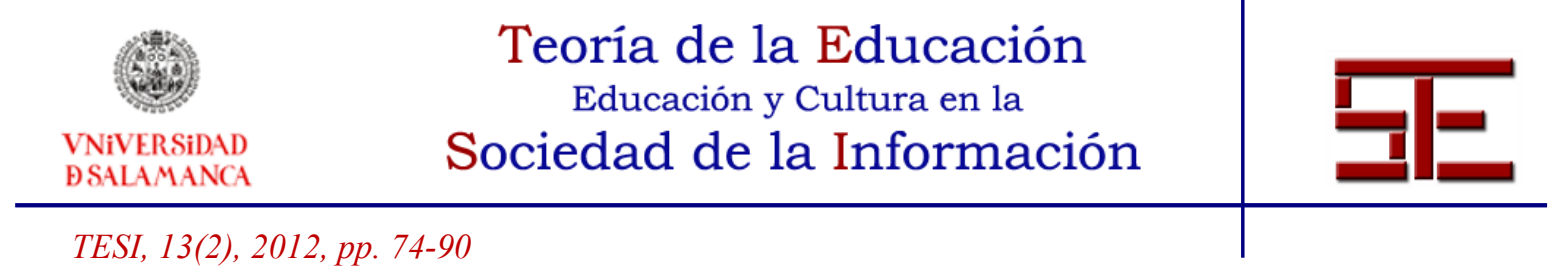

Moreno, I., Muñoz, L., Pittí, K., Quintero, J. y Serracin, J. (2011). Robótica Educativa como herramienta de enseñanza-aprendizaje en colegios secundarios. 6ta. Conferencia Ibérica de Sistemas y Tecnologías de la Información. Chaves, Portugal.

Olaskoaga, K. (2009). La robótica como apoyo al aprendizaje. Extraído el 12, de abril, 2011, de http://lrobotikas.net/es/proyectos-educativos/54-general/85-la-robotica-comoapoyo-al-aprendizaje.

Pisciotta, M., Vello, B., Bordo, C., Morgavi, G. (2010). Robotic Competition: A Classroom Experience in a Vocational School. En 6th WSEAS/IASME International Conference on Educational Technologies (EDUTE '10), pp. 151-156.

Pittí, K., Curto, B. \& Moreno, V. (2010). Experiencias construccionistas con robótica educativa en el Centro Internacional de Tecnologías Avanzadas. TESI. (11)1, 310-329 Extraído el 15 de mayo, 2012, de

http://campus.usal.es/\%7Erevistas_trabajo/index.php/revistatesi/article/view/6294/6307.

Pozo, E. G. (2005). Técnicas para la Implementación de la Robótica en la Educación Primaria. Recuperado el 10 de mayo de 2011, de Complubot: http://complubot.educa.madrid.org/actividades/inrerdidac_robotica_primaria.pdf.

Ruiz-Velasco, E. (2007). Educatrónica: innovación en el aprendizaje de las ciencias y la tecnología. Buenos Aires: Editorial Díaz de Santos, S.A.

Savage, T., Sánchez, A. , I., O’Donnell, F. \& Tangney, B. (2003). Using Robotic Technology as a Constructionist Mindtool in Knowledge Construction. En Proceedings of the The 3rd IEEE International Conference on Advanced Learning Technologies (ICALT'03).

Zúñiga, A. L. (2006). Fundacion Omar Dengo. Recuperado el 10 de abril de 2011, de http://www.fod.ac.cr/robotica/descargas/roboteca/articulos/2009/motorinnova_corto.pdf

Para citar el presente artículo puede utilizar la siguiente referencia:

Moreno, I., Muñoz, L., Serracín, J. R., Quintero, J., Pittí Patiño, K. y Quiel, J. (2012). La robótica educativa, una herramienta para la enseñanza-aprendizaje de las ciencias y las tecnologías. Revista Teoría de la Educación: Educación y Cultura en la Sociedad de la Información. 13(2), 74-90 [Fecha de consulta: dd/mm/aaaa].

http://campus.usal.es/ revistas_trabajo/index.php/revistatesi/article/view/9000/9245

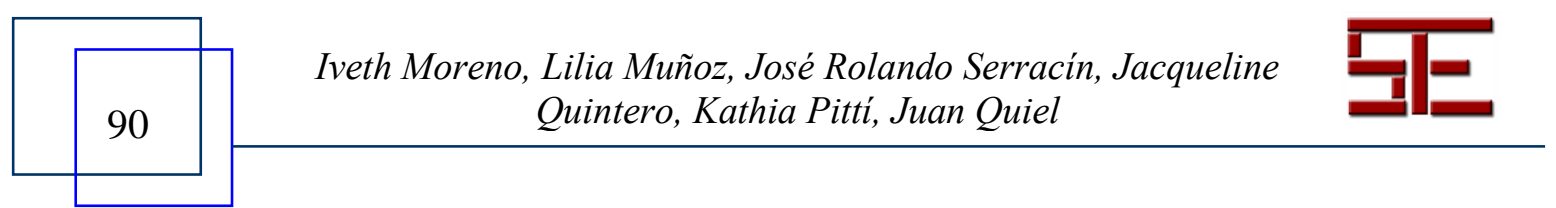

